\title{
Eau colorée à Lingulodinium polyedrum (Stein) Dodge, dans une zone aquacole du littoral du Doukkala (Atlantique marocain)
}

\section{Lingulodinium polyedrum (Stein) Dodge red tide in shellfish areas along Doukkala coast (Moroccan Atlantic)}

\author{
A. Bennouna a,b,*, B. Berland ${ }^{\text {c }}$, J. El Attar ${ }^{\text {a }}$, O. Assobhei ${ }^{\mathrm{b}}$ \\ ${ }^{a}$ Institut national de recherche halieutique (INRH), Station de surveillance de la salubrité littorale, Oualidia, Maroc \\ ${ }^{\mathrm{b}}$ Faculté des sciences d'El Jadida, Maroc \\ ${ }^{\mathrm{c} C e n t r e ~ d ' o c e ́ a n o l o g i e ~ d e ~ M a r s e i l l e, ~ S t a t i o n ~ m a r i n e ~ d ' E n d o u m e, ~ r u e ~ d e ~ l a ~ B a t t e r i e-d e s-L i o n s, ~} 13007$ Marseille, France
}

Reçu le 11 décembre 2001; accepté le 24 avril 2002

\section{Résumé}

Une eau colorée à Lingulodinium polyedrum est survenue le long des côtes marocaines atlantiques en juillet 1999. Observée au début du mois dans la région de Kénitra, elle s'est étendue avec la dérive littorale jusqu'au sud de Safi et a touché, au passage, une zone soumise à une surveillance sanitaire. Cette zone comprend d'importants gisements naturels de moules et de palourdes, ainsi que les sites exploités d'Oualidia et de Sidi Moussa. Elle a fait l'objet d'un suivi bimensuel du phytoplancton durant un an ; toutefois, lors de l'efflorescence, une fréquence hebdomadaire a été adoptée. Des données hydrologiques et climatiques ont été associées aux dénombrements du phytoplancton afin d'identifier les causes du développement de cette efflorescence.

Cette efflorescence est apparue pour des températures des eaux superficielles, de $17-18{ }^{\circ} \mathrm{C}$ propices au désenkystement et au développement du dinoflagellé. Le schéma observé de deux à trois bandes parallèles à la côte suggère une agrégation du phytoplancton générée par des vagues internes engendrées par les vents modérés de direction et de vitesse constantes. De même, un changement des conditions climatiques est à l'origine de la dissipation de l'eau colorée. Il faut noter que l'efflorescence s'est toujours maintenue à distance des sites côtiers et des lagunes surveillés, les concentrations de Lingulodinium observées étaient au maximum de $79.10^{3}$ cell $1^{-1}$ dans la lagune de Sidi Moussa et, au minimum, variaient entre $1,6.10^{3}$ cell $1^{-1}$ et $18.10^{3}$ cell $1^{-1}$ dans les stations littorales.

La toxicité de type diarrhéique trouvée dans certains coquillages (moules, huîtres) ne peut être le fait de $L$. polyedrum mais plutôt d'espèces accompagnatrices telles Dinophysis acuminata et $D$. acuta que nous avons dénombrées.

Il semble pourtant nécessaire, étant données les controverses sur la toxicité de L. polyedrum et sa large implication dans des eaux colorées, de l'inclure parmi les espèces potentiellement nuisibles à surveiller le long des côtes marocaines. (C) 2002 Ifremer/CNRS/IRD/Éditions scientifiques et médicales Elsevier SAS. Tous droits réservés.

\begin{abstract}
A red tide of Lingulodinium polyedrum occured along the moroccan atlantic coast in july 1999. Observed in the beginning of the month in the Kenitra area, it spreads with the coastal drift as far as the south of safi, passing on its way upon a sanitary monitored area. This area which is submitted to an all year round bimonthly monitoring analysis of phytoplankton, contains significant natural beds of oysters, clams and mussels, as well as shellfish farms in Oualidia and Sidi Moussa lagoons. However, during this period of bloom, there was weekly monitoring of hydrologic and climatic data associated with phytoplankton analysis to identifiy the causes of this bloom.

This bloom occured in $17-18^{\circ} \mathrm{C}$ surface waters, temperatures which are favourable to dinoflagellate encystment and development. The observation of two to three colored strips parallel to the coast suggests phytoplankton aggregation generated by internal waves created by moderate winds blowing constantly in the same direction and at constant speed at the time of the bloom. A change of climatic conditions might also be the cause of the dispersal of the phenomenon. We remark that this exceptional bloom has never reached the coastal shellfish sites, nor the monitored lagoons. The maximum Lingulodinium concentration observed was $79.10^{3}$ cell $1^{-1}$ at Sidi Moussa lagoon and
\end{abstract}

\footnotetext{
* Auteur correspondant.

Adressee-mail : bennouna@hotmail.com (A. Bennouna).
} 
minimum concentrations ranged from $1,6.10^{3}$ cell $1^{-1}$ to $18.10^{3}$ cell $1^{-1}$ at coastal stations. The diarrheic toxicity found in the shellfish (mussels, oysters) cannot be imputed to L. polyedrum but to associated species such as Dinophysis acuminata and D. acuta which were present, though in low density in these waters.

However, taking into account the controversy on the L. polyedrum toxicity and its frequent involvement in red tides, it is necessary to include it among the potentially harmful species that should be monitored along Moroccan coasts. (C) 2002 Ifremer/CNRS/IRD/Éditions scientifiques et médicales Elsevier SAS. All rights reserved.

Mots clés: Eau colorée; Lingulodinum polyedrum; Toxicité; Maroc

Keywords: Red tide; Lingulodinium polyedrum; Toxicity; Morocco

\section{Introduction}

Bien que des efflorescences exceptionnelles existent depuis longtemps sur les côtes marocaines, les premières observations datent seulement de 1966. Au cours des deux dernières décennies, ces événements, toxiques ou non, sont de plus en plus fréquents. Sur la côte atlantique, ils apparaissent de manière cyclique, en général en fin d'été et au début de l'automne. Ils ont touché différents sites comme Larache, Kenitra, Rabat, Salé, Mohammedia, Rochesnoires, Casablanca, Safi, Essaouira, Agadir. Ils sont souvent accompagnés de cas d'intoxication humaine liés principalement à la consommation de moules (Mytilus edulis) mais aussi aux haricots de mer (Donax trunculus). Un programme de surveillance des efflorescences algales marines a donc été lancé en 1995.

Durant l'été 1999, un épisode d'eaux rouges de grande étendue a été observé au large de la côte atlantique allant de Kénitra à Agadir. L'espèce responsable était Lingulodinium polyedrum, espèce commune dans les régions tempérées, à l'origine de nombreux phénomènes d'eaux rouges. Cette espèce a parfois été associée à des événements toxiques ; toutefois sa toxicité reste controversée. Sur les côtes atlantiques marocaines, $L$. polyedrum n'avait jamais été observé jusqu'à cette date à de fortes densités. Sa présence avait, par contre, été noté en mars 1997 à Oualidia, à des concentra-

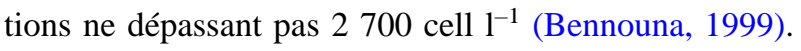

Dans ce travail, nous décrivons l'évolution temporelle et spatiale de cette eau rouge et discutons des causes de cette efflorescence en liaison avec les données hydroclimatiques. Nous étudierons également l'impact de cette efflorescence sur des sites littoraux comportant d'importants gisements naturels de moules et de palourdes ainsi que des parcs aquacoles (huîtres).

\section{Matériel et Méthodes}

\subsection{Présentation des sites d'étude}

La zone d'étude se situe le long des côtes atlantiques marocaines allant de Sidi Moussa à Essaouira Kdema sur environ $160 \mathrm{~km}$, avec sept stations littorales comprenant d'importantes moulières naturelles et deux stations dans des écosystèmes lagunaires (lagunes de Oualidia et de Sidi Moussa) qui sont des sites aquacoles exploités et une station plus au large (Fig. 1).

Les neuf premières stations sont étudiées dans le cadre du programme de surveillance des efflorescences assuré par la station de l'INRH à Oualidia, depuis 1997 pour les deux lagunes et 1999 pour les stations côtières. Les stations littorales (Jamâa Oueld Rhanem, Dar Lhamra, Lala Fatna, Cap Bedouza, Safi, Essaouira Kdema) sont situées le long d'une côte rocheuse, le plus souvent constituée par un système dunaire consolidé, dans la zone de déferlement des vagues. La lagune de Oualidia, allongée parallèlement à la côte, se situe à une distance de $76 \mathrm{~km}$ au sud d'El Jadida. Sa superficie totale est estimée à $4 \mathrm{~km}^{2}$. En moyenne, les profondeurs maximales et minimales sont respectivement de $5 \mathrm{~m}$ et de $1,5 \mathrm{~m}$. La lagune de Sidi Moussa, située à $40 \mathrm{~km}$ au nord de Oualidia présente une superficie de $2,5 \mathrm{~km}^{2}$. Les profondeurs varient entre $4 \mathrm{~m}$ et $1 \mathrm{~m}$. Quatre points de prélèvement par lagune ont été étudiés, près de parcs ostréicoles, ou des passes avec l'océan.

La station située au large, à plus de $200 \mathrm{~m}$ de la ligne de côte, se situe en face de la passe principale de la lagune de Oualidia. Elle a été échantillonnée dès le début de l'efflorescence.

\subsection{Caractéristiques hydroclimatiques des sites d'étude}

Le climat de la zone étudiée est aride à semi-aride. Des températures maximales de $40{ }^{\circ} \mathrm{C}$ en été, ont été relevées lorsque souffle un vent chaud, le Chergui. Mais, en général, la température atmosphérique moyenne journalière varie entre $21^{\circ} \mathrm{C}$ et $22^{\circ} \mathrm{C}$ en été, $14^{\circ} \mathrm{C}$ et $15^{\circ} \mathrm{C}$ en hiver.

Les vents sont sous l'influence de l'anticyclone des Açores. En période sèche, l'anticyclone engendre les alizés de direction NE à SO. En général, les vents dans la région sont constants, plus forts en été, faibles et intermittents en hiver, avec une répercussion sur la température de l'atmosphère et de l'eau de surface. Le régime hydrologique de cette côte est soumis essentiellement au rythme des marées semi-diurnes et à l'action de remontées d'eau qui affectent toute la zone, surtout en été. Cette zone littorale est caractérisée par un fort courant de dérive se dirigeant vers le sud. 


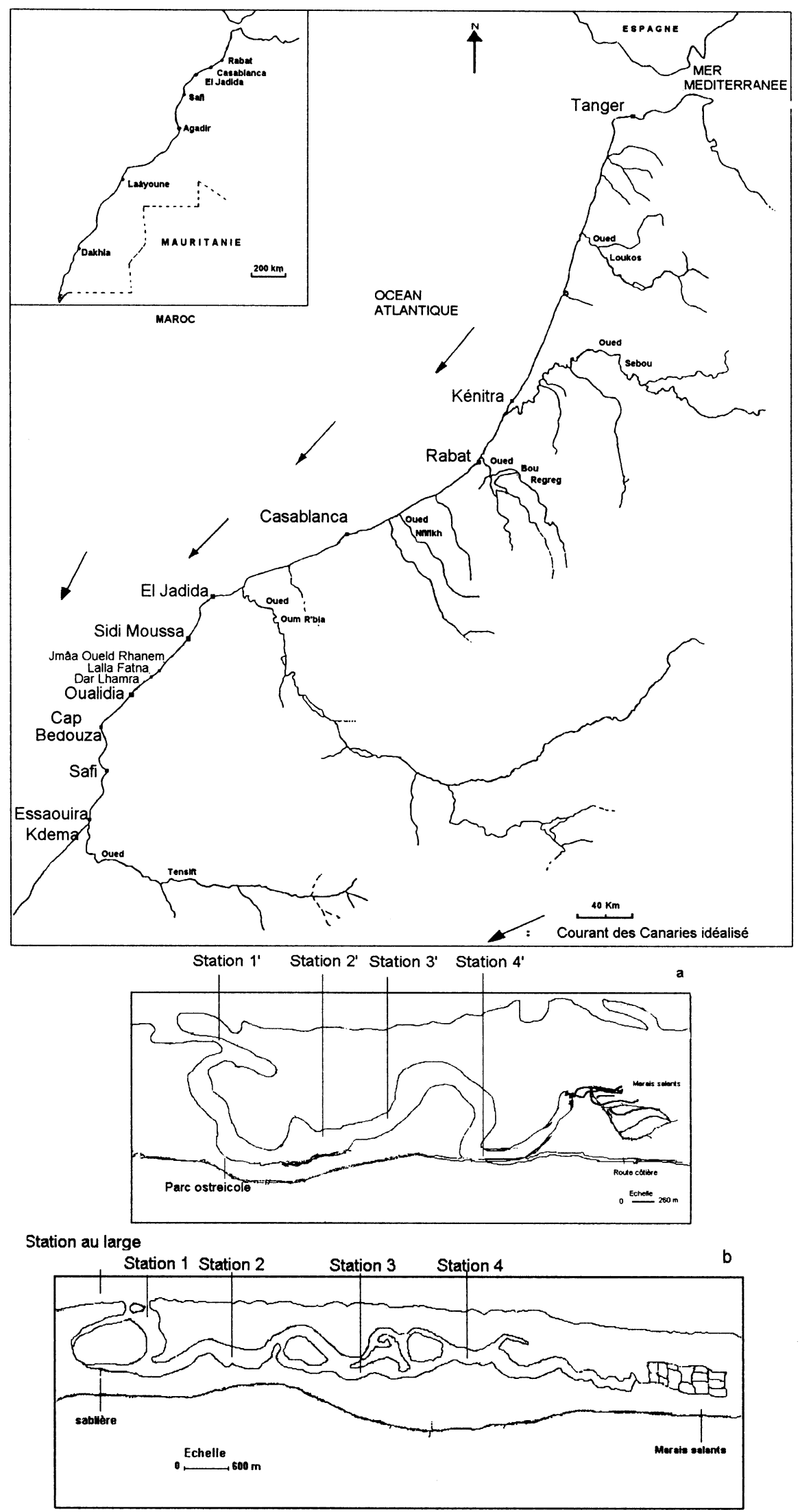

Fig. 1. Localisation des stations d'échantillonnage, a : lagune de Sidi Moussa ; b : lagune de Oualidia. Location of sampling stations, a: Sidi Moussa lagoon; b: Oualidia lagoon. 


\subsection{Collecte du phytoplancton et des coquillages}

La collecte du phytoplancton a été effectuée en surface à marée haute, avec une fréquence hebdomadaire lors de l'efflorescence et bimensuelle en dehors de cette période. Certains échantillons ont été fixés au lugol pour une observation immédiate et d'autres au formol à $3 \%$ neutralisé pour une observation ultérieure au microscope électronique à balayage (MEB). Parallèlement aux analyses du phytoplancton, des prélèvements de coquillages (moules, huîtres et palourdes) ont été réalisés dans les mêmes sites à marée basse et envoyés pour analyse au laboratoire central de l'INRH à Casablanca. Les paramètres, température et salinité, sont mesurés in situ à l'aide d'un salinomètre de terrain WTW type LF 196. Les données météorologiques pour l'année 1999 ont été fournies par une station météorologique proche de Oualidia.

\subsection{Détermination et dénombrement du phytoplancton}

La détermination du phytoplancton a été effectuée par la méthode d' Utermöhl (1958) dans des cuves à sédimentation d'une capacité de 10 à $25 \mathrm{ml}$. Les échantillons observés au MEB, ont été traités selon le protocole décrit par Grzebyk et al. (1998).

\section{Résultats}

\subsection{Description de l'espèce responsable de l'eau colorée}

L'efflorescence est dûe à la prolifération du dinoflagellé Lingulodinium polyedrum (Stein) Dodge (1989). En effet, les observations au MEB (Figs. 3a, b, c, d, e, f), montrent que les cellules ont un aspect anguleux, avec des plaques thécales épaisses, présentant, suivant les sutures, des arêtes prononcées et des sculptures circulaires aux alentours des pores trichocystaires. Le cingulum est descendant et croisé (Fig. c). L'apex est tronqué. La tabulation épithécale est : Po, 3', 4a, 6'. 3' est une très petite plaque sur le côté droit du complexe du pore apical (Figs. d, e). C'est ce qui distingue L. polyedrum des autres espèces appartenant au genre Gonyaulax, dans lequel elle était placée auparavant (Dodge, 1989). Le sulcus est droit, large dans la partie postérieure (Fig. 3c).

C'est une espèce connue pour former des kystes de résistance sphériques, avec des projections épineuses, connues sous le nom de L. machaerophorum (Lewis et Burton, 1988). Dans nos échantillons cependant, nous avons observé, en fin de floraison, uniquement des formes nues, rondes, sans ornementation (Figs. 3g, h, i) qui correspondent aux kystes temporaires décrits par Marasovic (1993).

\subsection{Conditions hydroclimatiques}

Les conditions climatiques de l'année 1999 sont présentées sur la Fig. 2. La période avant et après la floraison (début mai-fin août) est caractérisée par :

- des précipitations journalières nulles (Fig. 2a), sauf début mai, où elles ne dépassent toutefois pas les $10 \mathrm{~mm}$;

- des températures atmosphériques moyennes journalières maximales par rapport au reste de l'année, oscillant entre 23 et $26^{\circ} \mathrm{C}$, avec une chute notable mais ponctuelle fin juin atteignant $14{ }^{\circ} \mathrm{C}$ suivie immédiatement d'un pic de $31^{\circ} \mathrm{C}$ le $1^{\text {er }}$ juillet (Fig. 2b). Au cours de la période de floraison, la température moyenne de l'air est restée relativement stable ;

- un rayonnement solaire journalier important lors de l'efflorescence, avec une moyenne oscillant entre 23 et 28-MJ m ${ }^{-2}$ (Fig. 2c). Ceci correspond à une importante insolation (d'environ 9 heures par jour) ;

- une mer calme avec une légère brise correspondant à des vitesses de vents, lors de la période de floraison, de 11 à $14 \mathrm{~km} \mathrm{~h}^{-1}$. Une élévation de la vitesse à $16,5 \mathrm{~km} \mathrm{~h}^{-1}$ est notée le 31 juillet, suivie d'une chute rapide le $1^{\text {er }}$ août correspondant à $3,9 \mathrm{~km} \mathrm{~h}^{-1}$ (Fig. $2 \mathrm{~d}$ );

- un marnage ne dépassant pas 1,6 $\mathrm{m}$ lors de la période de l'efflorescence, correspondant à une période de mortes-eaux (coefficients de marée entre 20 et 30) pendant laquelle les courants de marée sont minimaux, la stabilité des étales prolongée ;

- les températures moyennes des eaux de surface oscillent entre un minimum de $16,2^{\circ} \mathrm{C}$ au Cap Bedouza en mai et un maximum de $20,2^{\circ} \mathrm{C}$ en août dans la lagune de Oualidia ;

- les salinités restent stables aux alentours de 35 même dans les lagunes, reflétant bien l'influence des eaux océaniques et l'absence ou/et la faiblesse des apports d'eaux douces le long de cette zone côtière.

\subsection{Phytoplancton}

\subsection{1. Évolution spatio-temporelle de l'efflorescence (Fig. 4)}

Le laboratoire central de l'INRH à Casablanca a signalé, dès le 6 juillet, la présence de Lingulodinium polyedrum, dominant largement la microflore des eaux littorales entre Kénitra et Casablanca, au nord de la zone étudiée. À la même époque, dans cette dernière zone, aucune cellule de $L$. polyedrum n'a été observée, sauf à Lala Fatna où $3,2.10^{3}$ cell $1^{-1}$ ont été dénombrées. L'alerte étant donnée, la fréquence d'échantillonnage a été intensifiée. Toutefois, au large de Oualidia (à $200 \mathrm{~m}$ de la côte), les densités de $L$.

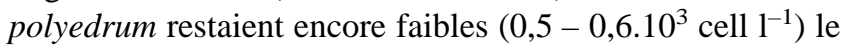
13 juillet. Dans les lagunes de Sidi Moussa et de Oualidia, l'apparition de cette espèce s'est produite entre le 14 et le 16 juillet avec des concentrations respectives de $0,16.10^{5}$ cell $1^{-1}$ à $7,3 \cdot 10^{3}$ cell $1^{-1}$. Elles étaient de l'ordre de $0,12 \cdot 10^{5}$ cell $1^{-1}$ au large de Oualidia le 20 juillet. Cette période 
a

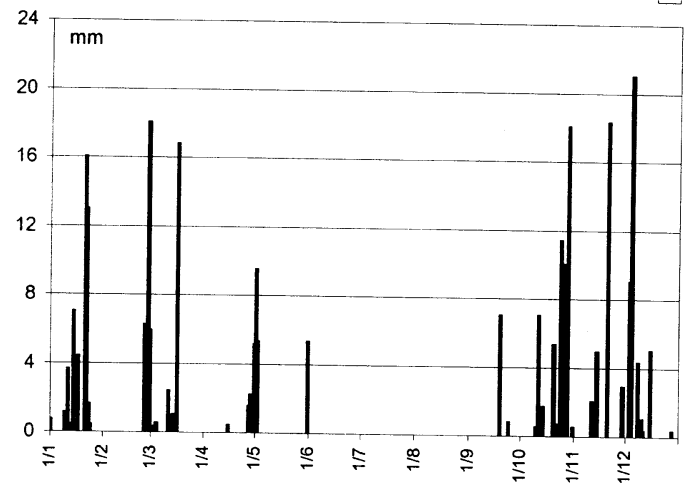

c

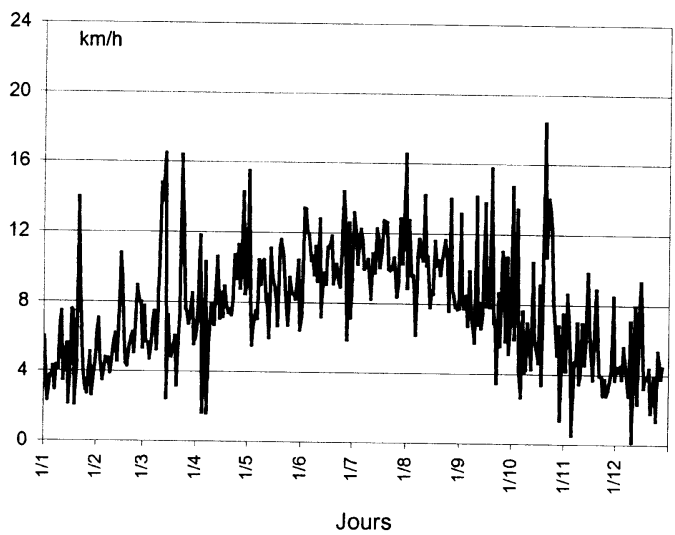

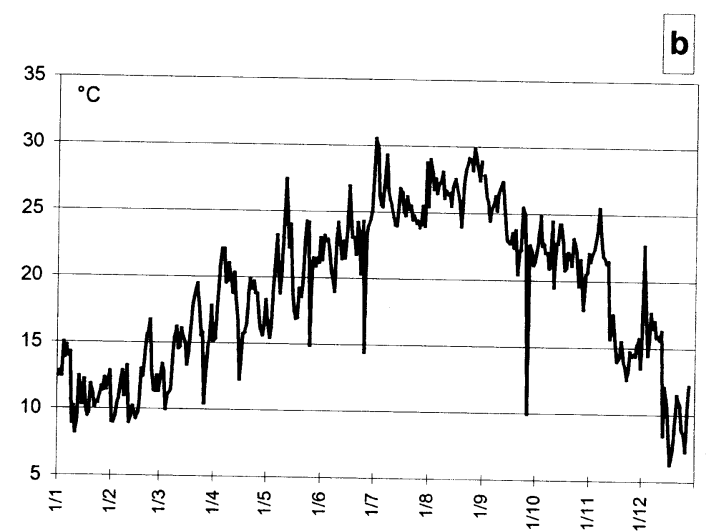

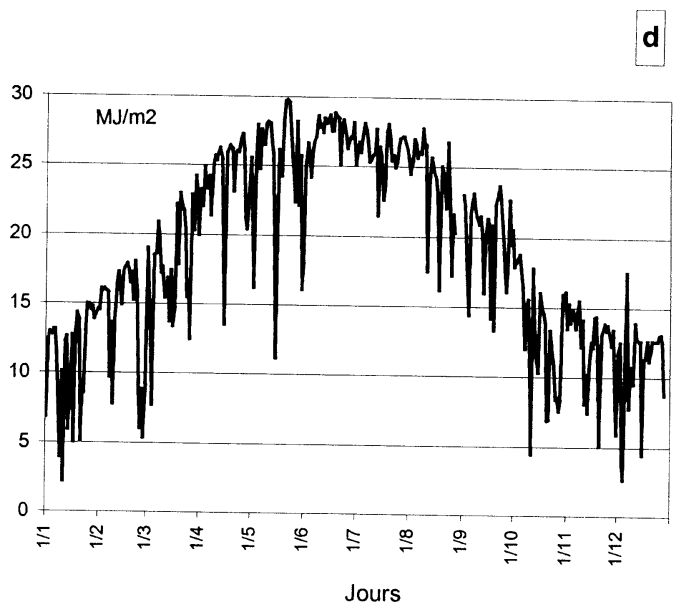

Fig. 2. Données météorologiques pour l'année 1999, a: pluviométrie, b: températures atmosphériques, c: vitesse des vents, d: rayonnement solaire.

Meteorological data for 1999 at the studied area, a: pluviometry, b: atmospheric temperature, c: wind speed, d: sun's radiation.

correspond à la phase d'initiation de l'efflorescence le long de la frange Kénitra - Essaouira Kdema, efflorescence qui s'est étendue lentement du nord vers le sud. La zone plus au sud (Essaouira et Agadir), n'était pas encore touchée à cette date.

$\mathrm{Au}$ début de la troisième semaine de juillet, par mer calme, une nappe d'eau rouge de plus en plus nette est observée au large des sites surveillés. Parallèle à la côte, elle forme trois bandes plus intensément colorées. Les prélèvements du 23 juillet à la station du large, située dans une des bandes, révèlent des concentrations maximales de L. polyedrum de l'ordre de $1,24.10^{6}$ cell $1^{-1}$, qui atteignent encore le 27 juillet $0,95 \cdot 10^{6}$ cell $1^{-1}$. Cette période correspond au « climax » de l'efflorescence à cette station. Pour les autres sites, on note à cette époque des valeurs nettement plus faibles que celles de la nappe du large. Les densités maximales sont de $0,79.10^{5}$ cell $1^{-1}$ dans la lagune de Sidi Moussa et de $0,27.10^{5}$ cell $1^{-1}$ dans celle de Oualidia. Elles varient entre $1,6.10^{3}$ cell $1^{-1}$ et $0,18.10^{5}$ cell $1^{-1}$ dans les stations littorales situées tant au nord qu'au sud de cette dernière lagune.

Début août, les concentrations chutent brutalement aussi bien au large qu'à la côte pour atteindre des densités de $1,4.10^{3}$ cell $1^{-1}$. Dans les lagunes toutefois, et plus particulièrement dans celle de Sidi Moussa, les densités demeurent légèrement plus élevées (maximum $2,7 \cdot 10^{3}$ cell $1^{-1}$ ). C'est la période de dispersion de l'efflorescence.

Cette floraison n'était pas, à proprement dit, monospécifique car L. polyedrum est accompagné par un autre dinoflagellé impliqué dans de nombreuses eaux colorées. Il s'agit de Prorocentrum micans, qui présente une évolution similaire. Cette co-évolution a été observée dans presque tous les sites étudiés.

\subsubsection{Phytoplancton total (Fig. 4)}

Dans les eaux de la lagune de Oualidia, les concentrations cellulaires phytoplanctoniques, toutes espèces confondues, présentent un pic post printanier début juin atteignant 

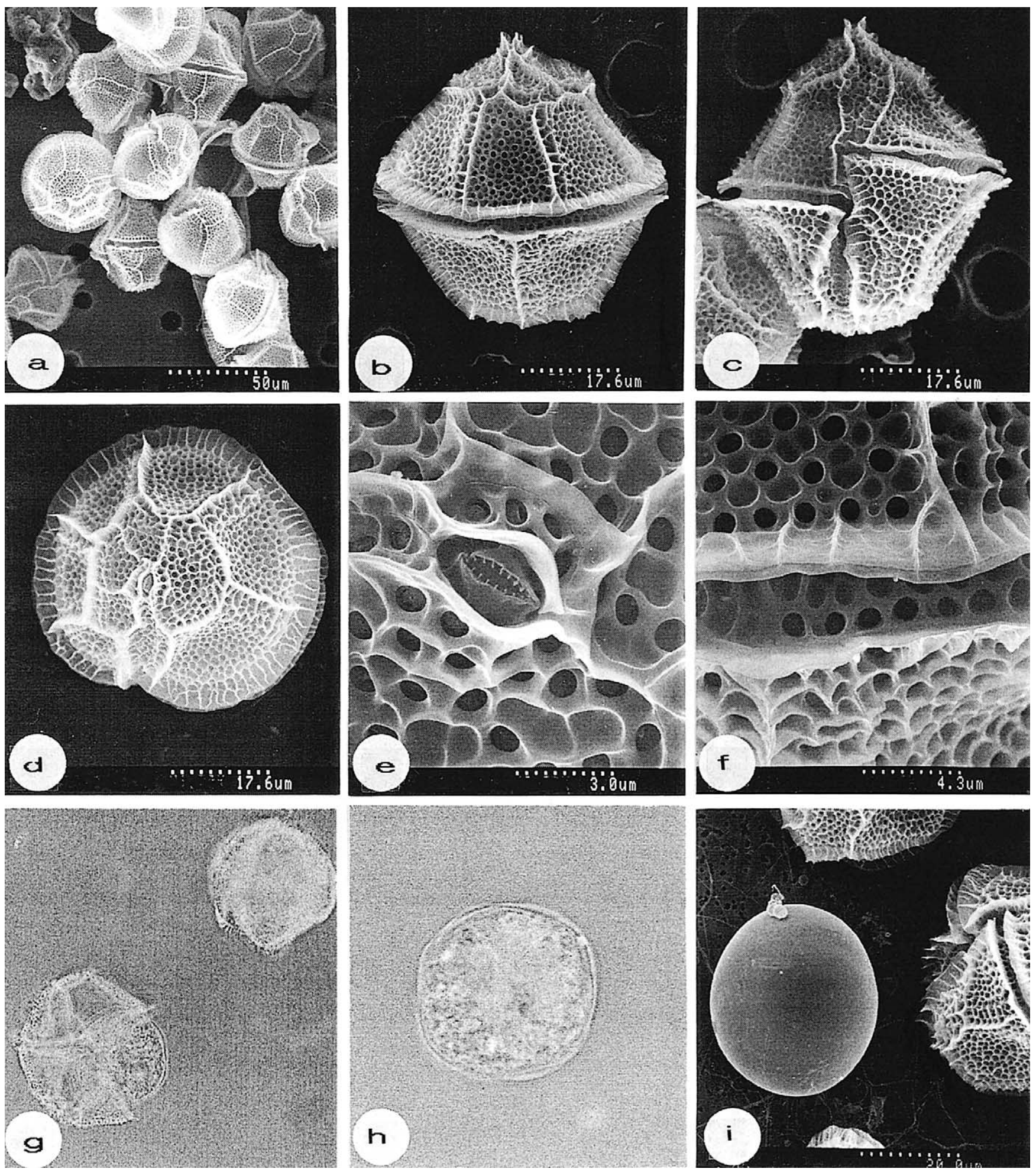

Fig. 3. a-i. Lingulodinium polyedra. a : amas de cellules végétatives de L. polyedrum (MEB) ; b : thèque vue dorsale (MEB); $\mathrm{c}:$ thèque vue ventrale (MEB) ; d : vue apicale (MEB) ; e : apex et détail de Po, 3' (MEB); f : cingulum (MEB); g : kyste temporaire émergeant d'une cellule en déhiscence ; h : kyste temporaire ; i : kyste temporaire (MEB).

a-i. Lingulodinium polyedrum. a: L. polyedra vegetative cell aggregates; b: dorsal view (SEM); c: ventral view (SEM); d: apical view (SEM); e: apex, and detail of Po,3' (SEM); f: cingulum (SEM); g: temporary cyst emerging from ecdysed cell; h: fully formed temporary cyst; i : temporary cyst (SEM).

$0,375.10^{6}$ cell $1^{-1}$, alors que ces concentrations ne dépassent pas $0,72.10^{5}$ cell $1^{-1}$ le 23 juillet, date correspondant au pic de L. polyedrum $\left(0,27.10^{5}\right.$ cell $\left.1^{-1}\right)$ dans cette lagune. Pour la lagune de Sidi Moussa, le pic des concentrations phytoplanctoniques a été atteint le 29 juin, il était de l'ordre de $0,344.10^{6}$ cell $^{-1}$, alors que ces densités étaient faibles, ne 

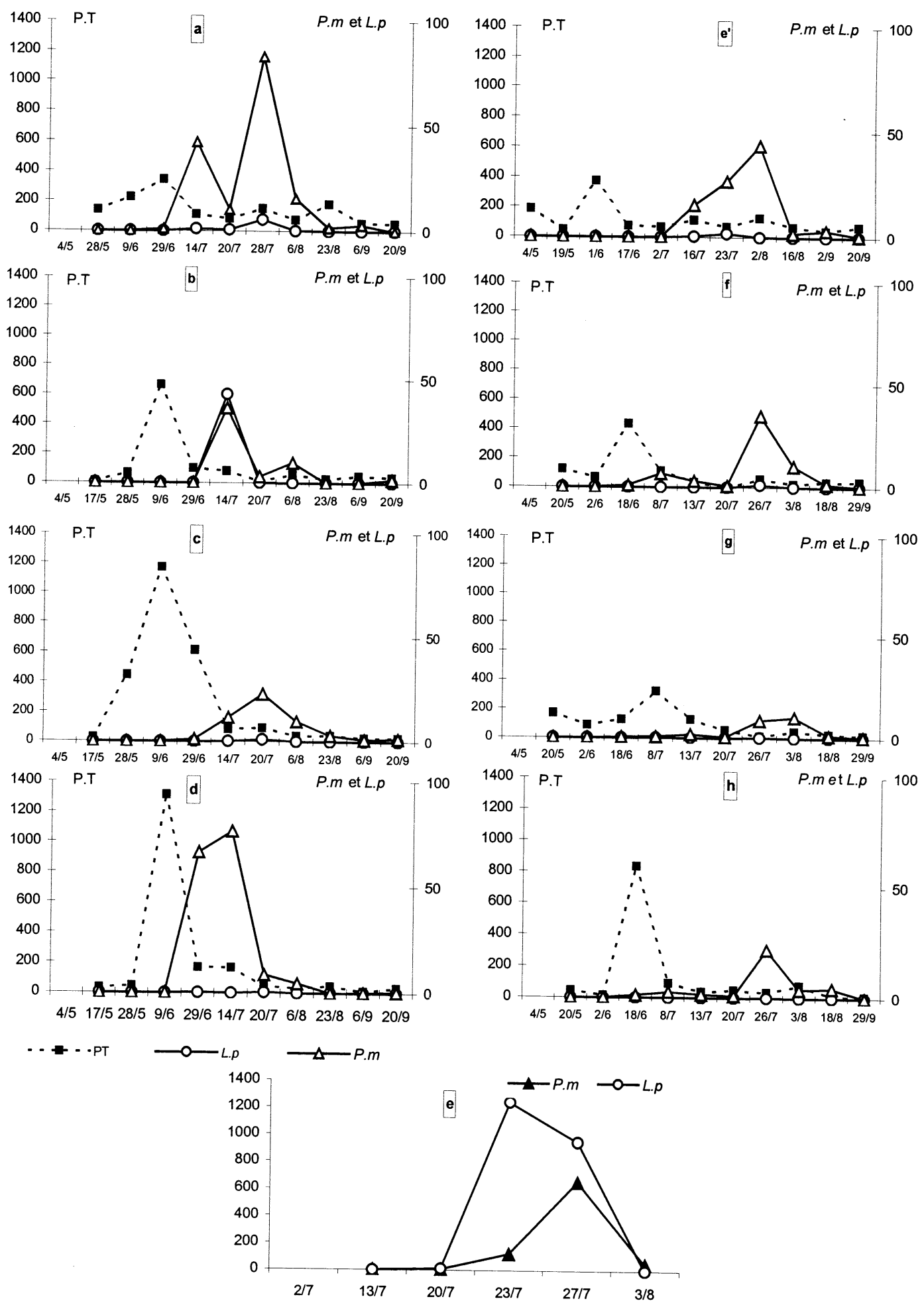

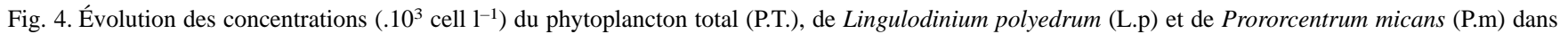
les eaux de surface, de mai à septembre 1999. a : Lagune de Sidi Moussa ; b : Jamâa Ouled Rhanem ; c : Dar Lhamra, d : Lala Fatna ; e : Large de Oualidia ; e' : Lagune de Oualidia ; f : Cap Bedouza ; g : Safi ; h : Essaouira Kdema.

Evolution of total phytoplankton, Lingulodinum polyedrum, Prorocentrum micans concentrations $\left(.10^{3}\right.$ cell $\left.1^{-1}\right)$ in surface waters during may to september 99. a: Sidi Moussa lagoon; b: Jamaa Ouled Rhanem; c: Dar Lhamra; d: Lala Fatna; e: Oualidia sea; e’: Oualidia lagoon; f: Cap Bedouza; g: Safi; h: Essaouira Kdema. 
dépassant pas un maximum de $0,153.10^{6}$ cell $1^{-1}$ le 28 juillet, lors du maximum de L. polyedrum $\left(0,79 \cdot 10^{5}\right.$ cell $\left.1^{-1}\right)$.

Dans la zone côtière, au nord de Oualidia, les concentrations phytoplanctoniques étaient maximales le 9 juin atteignant $1,2.10^{6}$ cell $^{-1}$ à Dar Lhamra. Elles demeurent faibles, à cette même station, lors du pic de l'efflorescence du large, ne dépassant pas $0,94.10^{5}$ cell $1^{-1}$. Au sud de Oualidia, on a relevé également ce pic post-printanier de la microflore correspondant à $0,84.10^{6}$ cell $1^{-1}$ à Essouira Kdema. Là encore, les concentrations chutent lors de l'efflorescence au large, vers des valeurs ne dépassant pas un maximum de $0,58.10^{5}$ cell $\mathrm{l}^{-1}$ enregistré au Cap Bedouza. La microflore était essentiellement constituée par deux groupes : les diatomées, le plus souvent dominantes tout au long de l'année et à un moindre degré, les dinoflagellés. Les silicoflagellés, les coccolithophorides et les cyanophycées restaient rares, représentés par une ou deux espèces et souvent en faibles densités (Fig. 5).

Dans les lagunes, uniquement pendant la période d'efflorescence à $L$. polyedrum (juillet - début août), les dinoflagellés dominaient avec des pourcentages atteignant 80 à $90 \%$ de la population totale. Pour les sites côtiers au nord de Oualidia, cette dominance était également marquée mais a débuté un peu plus tôt, à partir de juin et jusqu'en juillet, avec des pourcentages un peu moins élevés (40 à $80 \%$ de la microflore). Dans les eaux au sud de Oualidia, les diatomées continuaient à dominer les eaux superficielles à l'exception de fin juillet où les dinoflagellés étaient présents à des pourcentages allant de 40 à $60 \%$ de la population phytoplanctonique totale (Fig. 5). Les dinoflagellés présents dans les différentes stations étudiées, étaient surtout représentés par P. micans, rejoint et dépassé même par $L$. polyedrum lors de l'efflorescence.

\subsubsection{Espèces potentiellement nuisibles accompagnatrices de l'efflorescence}

Trois genres comportant des espèces potentiellement nuisibles ont été identifiés dans nos échantillons. Dinophysis était représenté surtout par $D$. acuminata et $D$. acuta, plus rarement par $D$. sacculus, D.rotundata et $D$. caudata. Toutes ces espèces confondues étaient présentes pendant l'efflorescence de $L$. polyedra à de faibles densités ne dépassant pas les 1000 cell $1^{-1}$.

Les espèces du genre Alexandrium sont absentes lors de l'efflorescence, mais elles sont observées avant et après l'efflorescence, à des concentrations toujours faibles ne

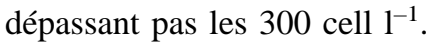

Le genre Gyrodinium est représenté essentiellement par Gyrodinium spiralee observé vers la fin de la floraison à des concentrations ne dépassant pas les $5.10^{3}$ cell ${ }^{-1}$.

\section{Discussion}

Certaines efflorescences à dinoflagellés sont initiées par suite de la germination de kystes enfouis dans les sédiments.
C'est notamment le cas de Lingulodinium polyedrum (Lewis et Burton, 1988 ; Marasovic, 1989) qui possède un kyste de résistance (L. machaerophorum). Le développement de cette efflorescence à $L$. polyedrum sur les côtes marocaines s'est effectué en période estivale, démarrant début juillet entre Kénitra et Casablanca, pour s'étendre lentement du nord vers le sud (région de Safi - Essaouira) avec la dérive littorale. Cette floraison est restée visible environ trois semaines.

Les températures des eaux superficielles des sites étudiés se situaient alors entre 17 et $18{ }^{\circ} \mathrm{C}$. D'après la littérature, c'est en période estivale qu'apparaissent en général les efflorescences à $L$. polyedrum. Ainsi, en mer Adriatique (Croatie), des efflorescences ont été observées de juin à septembre (Marasovic et al., 1995) se développant pendant trois semaines jusqu'à trois mois selon les conditions climatiques. Une durée par contre plus courte a été notée sur la côte atlantique (baie de Setubal, Portugal), ne dépassant pas dix jours (Palma et al., 2000). D'après Marasovic (1993), une température excédant $20^{\circ} \mathrm{C}$ est une condition cruciale pour l'initiation d'une efflorescence à $L$. polyedrum; cette température est également déterminante pour le maintien du bloom (Marasovic, 1990), toute diminution induisant son déclin. Pour Blanco (1990), la température a également une grande influence, mais le seuil de désenkystement se situe un peu plus bas ; ainsi en culture, à $15^{\circ} \mathrm{C}$, une nette diminution de la germination des kystes de $L$. polyedrum est observée par rapport à $18^{\circ} \mathrm{C}$. Les kystes utilisés dans cette dernière expérience provenaient d'une zone néritique de Galice située au nord ouest de l'Espagne, où les écarts annuels de température des eaux sont faibles $\left(12-18{ }^{\circ} \mathrm{C}\right)$. Dans la présente étude, l'efflorescence démarre en dehors de la zone étudiée, mais les températures des eaux superficielles de la côte nord marocaine sont proches de celles observées sur la côte du Doukkala. Nous sommes donc proches des conditions environnementales rencontrées par Blanco (1990). Le seuil thermique pour le déclenchement de la germination dépend de l'espèce étudiée et des caractéristiques environnementales (degré d'anoxie, nature du sédiment, intensité lumineuse). Pour Blanco (1990), l'intensité lumineuse semble ne pas avoir une grande influence sur la germination, par contre, cet auteur a montré que le stockage des kystes de $L$. polyedrum dans des conditions oxiques conduit pratiquement à l'inhibition de leur germination. Il y a peu de données quant à la nature des sédiments de la côte atlantique nord marocaine, sinon que le proche plateau continental est en général occupé par des sables grossiers bioclastiques. D'après le comportement sédimentaire des kystes, qu'il faut rapprocher de celui de particules fines inertes (diamètre de $60 \mu \mathrm{m}$ ), le site d'initiation devrait correspondre à une zone de sédimentation organique importante, telle que l'embouchure d'un estuaire (celui de Bouregreg) ou d'une zone portuaire (port de Kénitra). Notons également que le dénombrement du phytoplancton total a révélé un pic post-printanier correspondant à une floraison à diatomées, enregistré en juin dans les 
a

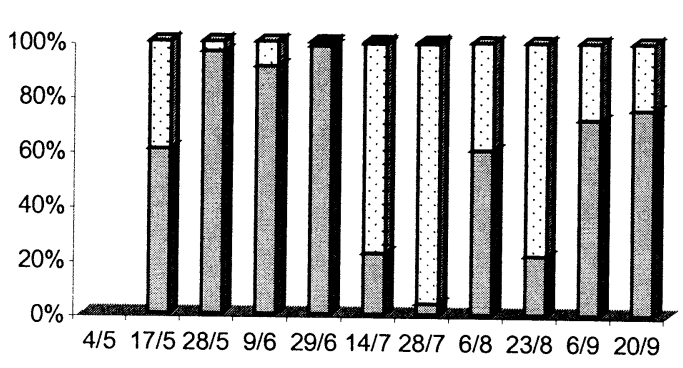

b

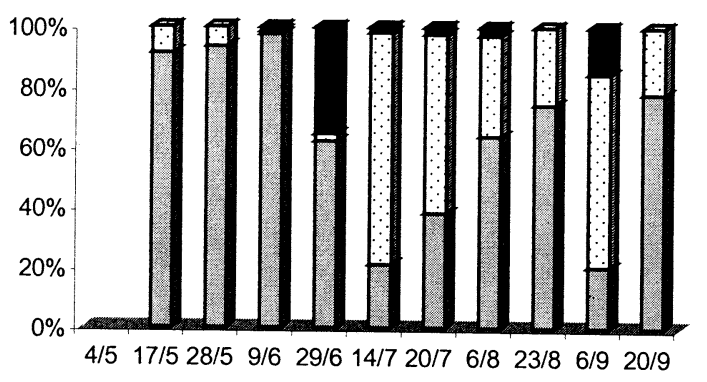

c

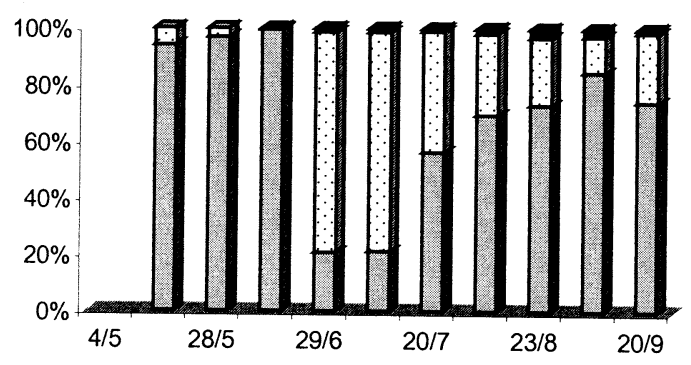

d

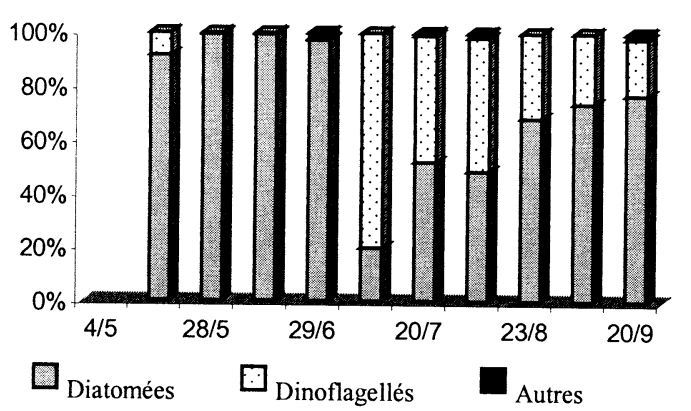

e

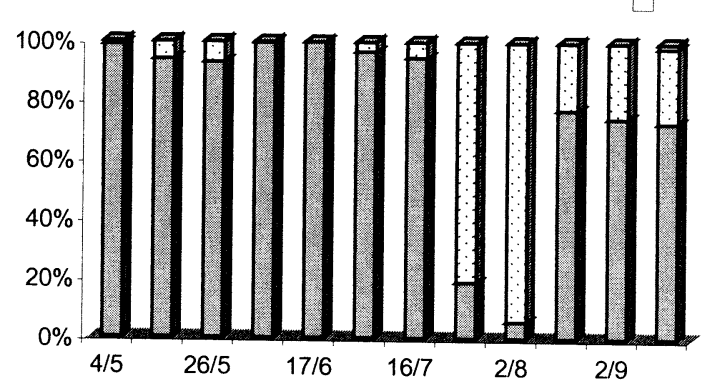

f

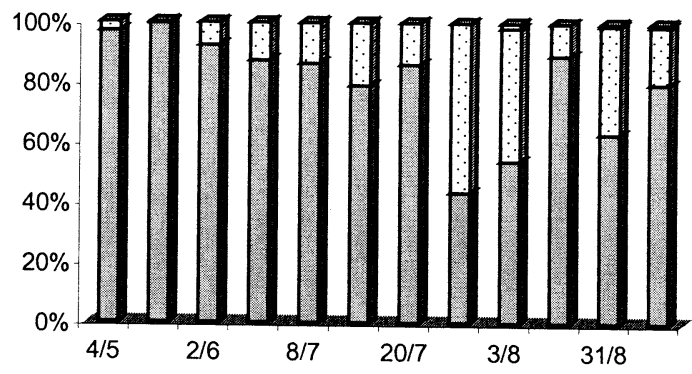

g

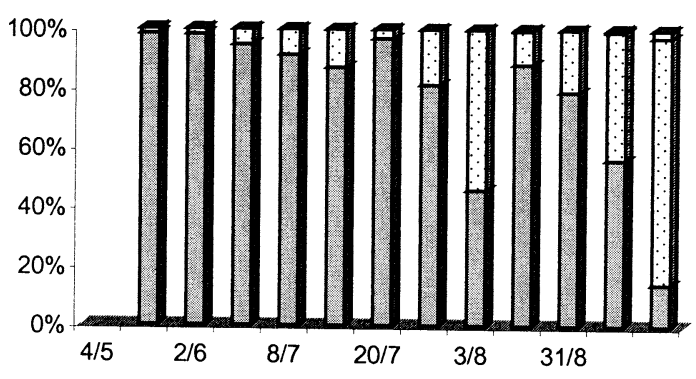

h

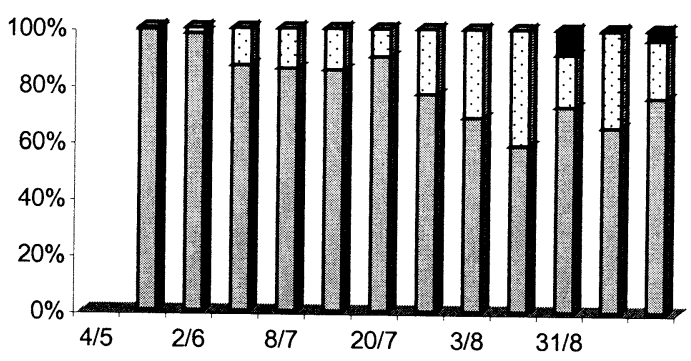

Fig. 5. Évolution des pourcentages des groupes phytoplanctoniques dans les eaux de surface. a : Lagune de Sidi Moussa ; b : Jamâa Ouled Rhanem ; $:$ Dar Lhamra ; : Lala Fatna ; e : Lagune de Oualidia ; f : Cap Bedouza ; g : Safi ; h : Essouiria Kdema.

Evolution of phytoplanktonic group percentages in surface waters a: Sidi Moussa lagoon; b: Jamaa Ouled Rhanem; c: Dar Lhamra; d: Lala Fatna; e: Oualidia lagoon; f: Cap Bedouza; g: Safi; h: Essouiria Kdema.

différents sites. C'est un phénomène observé de façon régulière le long de la côte atlantique (Bennouna, 1999). Ce développement phytoplanctonique devrait induire un certain degré d'anoxie dans les eaux plus confinées. Cependant, de tels processus sont moins ressentis dans les stations que nous avons étudiées, car il s'agit de zones sableuses 
ouvertes où déferlent les vagues, ou de lagunes soumises à des taux importants de renouvellement des eaux. Pour confirmer ces hypothèses, il faudrait établir une cartographie de la répartition des kystes appartenant à des espèces toxiques ou nuisibles afin de déterminer les zones de sédimentation sélective qui pourraient servir de réservoirs d'ensemencement.

L'efflorescence est devenue visible seulement au début de la troisième semaine de juillet avec des bandes rougeâtres parallèles à la côte; elles se sont formées au moment où, dans les stations côtières comme dans les lagunes, les densités phytoplanctoniques étaient faibles par rapport au mois précédent et les communautés constituées essentiellement de dinoflagellés. Ce schéma a déjà été décrit par plusieurs auteurs (Shanks, 1983, 1988 ; Franks, 1997). Ainsi, ce dernier a décrit des bandes d'eaux colorées à $L$. polyedrum, au niveau de La Jolla (Californie). Ces bandes, sont d'après lui, le résultat de l'interaction entre des particules activement mobiles, à phototropisme positif comme le sont les dinoflagellés et la dynamique physique locale. L'agrégation du phytoplancton en bandes peut être générée par des cellules de Langmuir provoquées par la force des vents (Evans et Taylor, 1980). Toutefois, nous pensons, pouvoir écarter cette origine, car selon les précédents auteurs, il s'agit d'un type de circulation à courte durée de vie, ne pouvant concentrer le phytoplancton que sur une distance de quelques mètres, ce qui n'est pas le cas dans l'efflorescence à $L$. polyedrum qui s'est étalée sur quelques centaines de kilomètres le long de la côte atlantique marocaine et qui a duré plus de trois semaines. Il faut donc rechercher une autre origine, comme l'existence de vagues internes à haute fréquence engendrées par des vitesses de vents modérées et stables (de l'ordre de $10 \mathrm{~km} \mathrm{~h}^{-1}$ ) (Taylor, 1987). D'après le modèle simple élaboré par Franks (1997), ce schéma spatial de constitution d'efflorescence par vagues internes ne correspond pas à un transport d'organismes mais plutôt à un processus transitoire d'accumulation: les organismes se concentrent au niveau des creux de ces vagues internes linéaires mais ne sont pas transportés avec la vague. Ceci peut expliquer le fait que les concentrations de $L$. polyedrum dans les stations côtières ainsi que dans les lagunes soient restées faibles par rapport à celles du large (huit à dix fois moins que celles du large), où la nappe dense est restée. De plus, dans les stations côtières, le degré de turbulence est plus élevé, ce qui peut également expliquer les densités faibles de $L$. polyedrum, espèce dont la croissance est inhibée même à des taux de turbulence relativement faibles (Gibson et Thomas, 1995). Dans les lagunes, une turbulence plus faible qu'à la côte, un taux de renouvellement des eaux minimum (période de mortes eaux) ainsi qu'une richesse nutritive un peu plus élevée que les eaux côtières Bennouna (1999), auraient permis un développement relatif du dinoflagellé, en particulier dans la lagune de Sidi Moussa $\left(1,7.10^{5}\right.$ cell $\left.1^{-1}\right)$.

Selon Taylor (1987), ces bandes générées par des vagues internes peuvent disparaître lorsque la vitesse des vents diminue ou augmente brusquement au-delà de $15 \mathrm{~km} \mathrm{~h}^{-1}$, conduisant à un état de turbulence. Lors de notre étude nous avons noté une augmentation de vitesse des vents fin juillet, suivie par une chute rapide le jour suivant. Ce changement météorologique peut être à l'origine de la dissipation de ces bandes d'efflorescence. Les kystes temporaires observés dans les échantillons de fin juillet reflètent des conditions défavorables du milieu, mais restent en densité trop faible pour être la cause du déclin de l'efflorescence.

La concomitance de $P$. micans et $L$. polyedrum notée dans nos échantillons, a également été signalée par Palma et al. (2000). D'après la littérature, il semble que ce soit souvent le cas $P$. micans est fréquemment retrouvée comme espèce co-dominante lors de blooms à dinoflagellés gonyaulacoïdes (Horstman, 1981). Cette co-existence correspond à des aptitudes comportementales proches, notamment des capacités de mobilité similaires chez ces espèces Taylor (1987).

Les analyses de toxines effectuées par test souris selon la méthode de Yasumoto et al. (1984), ont révélé, dès la deuxième semaine de juillet, la présence de toxines diarrhéiques (DSP), chez les moules et palourdes collectées au nord de Casablanca. Par contre, les coquillages provenant de la zone étudiée n'ont été contaminés qu'à partir de la troisième semaine de juillet, alors que ceux de la région plus au sud (Agadir) ne l'étaient pas encore. Nous constatons ainsi, une évolution de la contamination des bivalves concomitante à celle de l'efflorescence à $L$. polyedrum, ce qui pourrait laisser supposer la participation de cette efflorescence à la production de toxines DSP comme l'ont suggéré Tahri Joutei et al. (2000).

De fait, l'espèce $L$. polyedrum, responsable de nombreuses eaux rouges sans effets nuisibles ou à l'origine d'anoxie du milieu, a été à plusieurs reprises associée à des événements toxiques (Tableau 1). Ainsi, Schradi et Bliss (1962) ont rapporté un effet toxique sur les souris lors d'un test de dépistage de toxines PSP. Toutefois, la présence de ces toxines a été mise en question par plusieurs auteurs : le temps de latence long qui précédait la mort des souris, ne correspondait pas aux symptômes toxiques de la PSP. Plus tard, Bruno et al. (1990), par des bioessais, ont montré la présence de PSP chez un bloom de L. polyedrum, mais des analyses par HPLC n'ont détecté qu'une infime quantité de toxines PSP, nettement insuffisante pour être à l'origine de la toxicité observée chez les souris. Ces auteurs ont donc supposé qu'avec les PSP, d'autres toxines étaient produites par $L$. polyedrum. Cette conclusion a été reprise plus tard par Tubaro et al. (1998) et Draisci et al. (1999), qui ont identifié ces autres toxines comme étant des yessotoxines. La production de ces toxines par des efflorescences à $L$. polyedrum bien que fortement probable reste à être confirmée en culture pure comme cela l'a été chez une espèce voisine Gonyaulax grindleyi (Protoceratium reticulatum) (Seamer et al., 2000).

Des analyses par HPLC/SM réalisées par Taleb et Hummert (2000), sur certains échantillons de moules que nous 
Tableau 1

Historique des eaux colorées à Lingulodinium polyedrum dans le monde et évènements toxiques associés.

Table 1

History of Lingulodinium polyedrum red tides in the world and associated toxic events.

\begin{tabular}{|c|c|c|c|}
\hline Sites & Toxines & Commentaires & Références \\
\hline Sud de la Californie & PSP & Effet toxique sur les souris & (Schradie and Bliss, 1962) \\
\hline Golfe de Californie & Absence & $\begin{array}{l}\text { L.polyedrum est toujours dominante dans la population } \\
\text { phytoplanctonique }\end{array}$ & (Blasco, 1977) \\
\hline Port de Pula (Yougoslavie) & Absence & $\begin{array}{l}\text { Mortalité de poissons liée à l'anoxie du milieu, } \\
\text { la toxicité de L.polyedrum n'et pas prouvée }\end{array}$ & (Maretic et al., 1978) \\
\hline Nord Ouest Adriatique (Italie) & Absence & Mortalité de poissons liée à l'anoxie du milieu & (Boni, 1983) \\
\hline Émilie Romagne (Italie) & PSP & Mortalité de poissons liée à l'anoxie du milieu & (Fortuna et al., 1985) \\
\hline Sud de la Californie & Absence & & (Goodman et al., 1984) \\
\hline Est de la Méditerranéenne & Ichtyotoxines & $\begin{array}{l}\text { L.polyedrum est suspectée dans la production } \\
\text { d'ichtyotoxines, impliquées dans la mortalité d'huître } \\
\text { en } 1977\end{array}$ & (Lassus, 1988) \\
\hline $\begin{array}{l}\text { Baie de Kastela (Yougoslavie) } \\
\text { Mer Adriatique }\end{array}$ & Absence & Mortalité de poissons liée à l'anoxie du milieu & $\begin{array}{l}\text { (Marasovic and Pucher-Petkovic, } 1987 \text {; } \\
\text { Marasovic, 1989, 1990) }\end{array}$ \\
\hline Mer Adriatique (Italie) & PSP & Possibilité de présence d'autres toxines & (Bruno et al., 1990) \\
\hline Mer Adriatique & Homo YTX & $\begin{array}{l}\text { L.polyedrum est associée à des évènements de toxicités } \\
\text { L.polyedrum pourrait produire les yessotoxines }\end{array}$ & $\begin{array}{l}\text { (Honsell et al., 1992) } \\
\text { (Draisci et al., 1999) }\end{array}$ \\
\hline Côte de Chine & Absence & L.polyedrum est identifiée comme une espèce toxique & (Tseng et al., 1993 ; Mingyuan et al., 1997) \\
\hline Mer Adriatique & Homo YTX & L.polyedrum pourrait produire l'homoyessotoxine & (Tubaro et al., 1998 ; Yasumoto and Satake, 1998) \\
\hline Côtes Libanaises & Absence & & (Abboud-Abi Saab and El Bakht, 1998) \\
\hline Baie de Setubal (Portugal) & Absence & & (Palma et al., 2000) \\
\hline
\end{tabular}

avons collectés lors de cette efflorescence ont révélé la présence de quantités importantes d'acide okadaïque (AO) et de Dinophysis-toxine 2 (DTX-2). L'acide okadaïque est généralement produit par des espèces appartenant aux genres Prorocentrum (particulièrement $P$. lima) et Dinophysis. La Dinophysis-toxine 2 a été trouvée essentiellement chez D. acuta (Vale et al.,1997 ; Frenandez et al., 2000). Or précisément, parmi les espèces nuisibles accompagnant cette efflorescence, nous avons relevé la présence de Dinophysis dont $D$. acuminata et $D$. acuta.

\section{Conclusion}

La côte atlantique marocaine a donc connu pour la première fois durant l'été 1999, une efflorescence à $L$. polyedrum de grande ampleur, dans la mesure où elle a touché plus de $200 \mathrm{~km}$ de côte. Toutefois, les densités phytoplanctoniques atteintes sont restées modérées par rapport à certaines efflorescences de cette espèce observées dans d'autres mers (Adriatique notamment). Cette apparition a été sans doute favorisée par des conditions climatiques et météorologiques propices à la germination des kystes et par la formation de vents, soufflant à vitesse modérée dans une direction constante, qui ont pu engendrer cette agrégation d'organismes mobiles. De même c'est un changement brusque de la vitesse des vents qui a sans doute été à l'origine du déclin de cette efflorescence.

Dans le cas présent, l'efflorescence s'est maintenue au large sans ganger le rivage. Les sites côtiers ouverts et agités ont donc été peu touchés, pourtant les coquillages qu'ils abritaient, présentaient des taux de toxines DSP tels qu'une interdiction de collecte et d'exploitation a été prononcée. Nous pensons toutefois que $L$. polyedrum ne peut être à l'origine de cette toxicité de type diarrhéique (DSP) et ce pour plusieurs raisons : - la faible densité de cellules de $L$. polyedrum au niveau des moulières naturelles et dans les lagunes - la non présence de yessotoxines seules toxines susceptibles d'être produites par cette espèce, toxines qui provoquent des symptômes chez la souris différents de ceux provoqués par les toxines diarrhéiques - la présence de Dinophysis spp. dont $D$. acuminata et $D$. acuta producteurs de toxines diarrhéiques, qui sont sans doute à l'origine de la toxicité. Il semble pourtant nécessaire, étant données les controverses sur la toxicité de L. polyedrum et sa large implication dans des eaux colorées de l'inclure parmi les espèces potentiellement nuisibles à surveiller le long des côtes marocaines. La recherche des foyers d'enkystement et désenkystement, ainsi que la mise en culture de certaines souches s'avèrent primordiales pour une meilleure connaissance de la physiologie, de l'écologie et surtout la toxicité de cette espèce dans le but d'une meilleure prévention de ses efflorescences sur nos côtes.

\section{References}

Abboud-Abi Saab, M., El Bakht, Y., 1998. Dominant and potentially toxic microalgae in lebanese coastal waters. In: Reguera, et al. (Eds.), Harmful algae, Inter. Oceanog. Comm. UNESCO. pp. 92.

Bennouna, A., 1999. Étude du phytoplancton nuisible et de son environnement dans la lagune de Oualidia et de Sidi Moussa, thèse de $3^{\mathrm{e}}$ cycle. Université Chouaïb Doukkali, pp. 153.

Blanco, J., 1990. Cyst germination of two dinoflagellate species from Galicia (NW Spain. Sci. Mar. 54, 287-291.

Blasco, D., 1977. Red tide in the upwelling region of Baja California. Limnol. Oceanogr. 22, 255-263.

Boni, L., 1983. Red tides of the coast of Emilia Romagna (north- western Adriatic sea) fromto1982. Info. Bot. Ital. 15, 18-24. 
Bruno, M., Gucci, P.M.B., Pierdominici, E., Ioppolo, A., Volterra, L., 1990. Presence of saxitoxin in toxic extracts from Gonyaulax polyedra. Toxicon. 28, 113-116.

Dodge, J.D., 1989. Some revisions of the family Gonyaulacaceae (Dinophyceae) based on a scanning electron microscope study. Botanica Marina 32, 275-298.

Draisci, R., Ferretti, E., Palleschi, L., Marchiafava, C., Poletti, R., Milandri, A., Ceredi, A., Pompei, M., 1999. High levels of yessotoxin in mussels and presence of yessotoxin and homoyessotoxin in dinoflagellates of Adriatic sea. Toxicon. 37, 1187-1193.

Evans, G.T., Taylor, F.J.R., 1980. Phytoplankton accumulation in Langmuir cells. Limnol. Oceanogr. 25, 840-845.

Fernandez, M.L., Reguera, B., Martinez, A., 2000. Toxinology and toxin content of Dinophysis acuminata, D. acuta and D. caudata from the Galician rias bajas. Abstracts of $9^{\text {e }}$ Conf. Inter. of Harmful algal bloom 2000. pp. 116.

Fortuna, S., Volterra, L., Spano, A.M., Michalek, H., 1985. Ricerca di neurotossine nelle alghe e nei mitili raccolti durante la "Marea rossa" nell'estate 1984 sul littorale Romagnolo. Ann. Ist. Super. Sanità 21, 349-356.

Franks, P.J.S., 1997. Spatial patterns in dense algal blooms. Limnol. Oceanogr. 42, 1297-1305.

Gibson, C.H., Thomas, W.H., 1995. Effects of turbulence intermittency on growth inhibition of a red tide dinoflagellate, Gonyaulax polyedra. J. Geophysis. Res. 100, 24841-24846.

Goodman, D., Eppley, R.W., Reid, F.M.H., 1984. Summer phytoplankton assemblages and their environmental correlates in the Southern California Bight. J. Mar. Res. 42, 1019-1049.

Grzebyk, D., Sako, Y., Berland, B., 1998. Phylogenetic analysis of nine species of Prorocentrum (Dinophyceae) inferred from $18 \mathrm{~S}$ ribosomal DNA sequences, morphological comparisons and description of Prorocentrum panamensis sp. J. Phycol. 34, 1055-1068.

Honsell, G., Boni, L., Cabrini, M., Pompei, M., 1992. Toxic or potentially toxic dinoflagellates from the Northern Adriatic sea. In: Viviani, R., Marchetti, R., Vollenweider, R.A. (Eds.), Marine coastal eutrophication. Elsevier, Amsterdam, pp. 107-144.

Horstman, D.A., 1981. Reported red-water outbreaks and their effects on fauna of the west and south coasts of South Africa,1959-1980. Fish. Bull. Soc. S. Afr. 15, 71-88.

Lassus, P., 1988. Plancton toxique et plancton d'eaux rouges sur les côtes européennes. IFREMER, Centre de Brest, pp. 111.

Lewis, J., Burton, P., 1988. A study of newly excysted cells of Gonyaulax polyedra (Dinophyceae) by electron microscopy. Br. Phycol. J. 23, 49-60.

Marasovic, I., 1989. Encystement and excystment of Gonyaulax polyedra during a red tide. Estuar. Coast. Shelf Sci. 28, 35-41.

Marasovic, I., 1990. Temperature Initiation factor of red tide bloom in the Kastela bay (Adriatic Sea, Yougoslavia). Rapp. Comm. Int. Mer Médit 32 (211).

Marasovic, I., 1993. Preliminary observations on the relationship between temporary and resting cysts of Lingulodinium polyedra (Stein) Dodge. In: Smayda, T.J., Shimizu, Y. (Eds.), Toxic phytoplankton bloom in the sea. Elsevier, New York, pp. 139-142.

Marasovic, I., Nincevic, Z., Odzak, N., 1995. The effect of temperature on blooms of Lingulodinium polyedra and Alexandrium minutum in Kastela bay. In: Lassus, et al. (Eds.), Harmful marine blooms. pp. 187-192.
Marasovic, I., Pucher-Petkovic, T., 1987. Ecological observations in locally limited summer bloom. The effects of pollution of marine ecosystems, FAO, UNEP. Fish. Rep, 352, pp. 167-174.

Maretic, Z., Poled, I., Zekic, R., Bujan, M., 1978. Red tide due to dinoflagellates in the harbour of Pula. Period. Biol. 80, 153-159.

Mingyuan, Z., Ruixiang, L., Xueyan, M., Rubao, J., 1997. Harmful algal blooms in China Seas. Ocean. Res. 19, 173-184.

Palma, A.S., Ferreira, A.A., Sampayo, M.A., Moita, M.T., Rosa, T., 2000. On a Lingulodinium polyedra bloom in the Setubal bay, Portugal. Abstracts of $9^{\mathrm{e}}$ Conf. Inter. Harmful algal bloom, 2000. pp. 191.

Seamer, C., Gordon, M., Mackenzie, L., Truman, P., 2000. The production of yessotoxin by Protoceratium reticulatum. Abstracts of $9^{\mathrm{e}}$ Conf. Inter. Harmful algal bloom, 2000. pp. 217.

Shanks, A.L., 1983. Surface sliks associated with tidally forced internal waves may transport pelagic larve of benthic invertebrates and fishes shoreward. Mar. Ecol. Prog. Ser. 13, 311-315.

Shanks, A.L., 1988. Further support for the hypothesis that internal waves can cause shoreward transport of larval invertebrates and fish. Fish. Bull. 86, 703-714.

Schradie, J., Bliss, C.A., 1962. Cultivation and toxicity of Gonyaulax polyedra. Lloydia 25, 214-221.

Tahri Joutei, L., Berraho, A., Orbi, A., 2000. Harmful algae blooms and the upwelling regime in atlantic costal waters of Morocco. Abstracts of $9^{\mathrm{e}}$ Conf. Inter. Harmful algal bloom, 2000. pp. 145.

Taleb, H., Hummert, C., 2000. Okadaïc acid and dinophysistoxine-2 in Morocco. Abstracts of $9^{\text {e }}$ Conf. Inter. Harmful algal bloom, 2000. pp. 230.

Taylor, F.J.R., 1987. Ecology of dinoflagellates, General and marine ecosystems. In: Taylor, F.J.R. (Ed.), Biology of dinoflagellates. pp. 399-502.

Tseng, C.K., Zhou, M.J., Zou, J.Z., 1993. Toxic phytoplankton studies in China. In: Smayda, T.J., Shimizu, Y. (Eds.), Toxic phytoplankton Bloom in the sea. Elsevier, New York, pp. 347-352.

Tubaro, A., Sidari, L., Della Loggia, R., Yasumoto, T., 1998. Occurrence of yessotoxin- like toxins in phytoplankton and mussels from northern Adriatic sea. In: Reguera, et al. (Eds.), Harmful algae, Inter. Oceanog. Comm. UNESCO. pp. 470-472.

Utermöhl, H., 1958. Zur Vervollkommung der quantitativen phytoplancton. Methodik. mitt. int. Ver. Theor. Angrevo. Limnol. 9, 1-38.

Vale, P., Sampayo, M.A.M., Quilliam, M.A., 1997. DSP complex toxin profiles relation with Dinophysis spp. occurrence and domoic acid confirmation by LC-MS in portuguese bivalves. In: Reguera, et al. (Eds.), Harmful algae, Inter. Oceanog. Comm. UNESCO. pp. 503-506.

Yasumoto, T., Murata, M., Oshima, Y., Matsumoto, C.K., Clardy, J., 1984. Diarrethic shellfish poisoning. In: Ragelis, E.P. (Ed.), Seafood toxins, 262. American Chemical Society Sump, Washington, pp. 207-214.

Yasumoto, T., Satake, M., 1998. New toxins and their toxicological evaluations. In: Reguera, et al. (Eds.), Harmful algae, Inter. Oceanog. Comm. UNESCO. pp. 461-464. 